\title{
Associative photoproduction of charmed particles near threshold
}

\author{
Michail P. Rekalo * \\ Middle East Technical University, Physics Department, Ankara 06531, Turkey \\ Egle Tomasi-Gustafsson \\ DAPNIA/SPhN, CEA/Saclay, 91191 Gif-sur-Yvette Cedex, France
}

(December 7, 2018)

\begin{abstract}
We calculate the cross section and the beam asymmetry for exclusive photoproduction of charmed particles near threshold $\left(\gamma+p \rightarrow \Lambda_{c}^{+}+\overline{D^{0}}\right)$, in the framework of an effective Lagrangian model. We discuss the sensitivity of these observables to the magnetic moment of the $\Lambda_{c}$ baryon and of the coupling constant in the $p \Lambda_{c} D$ vertex. We show that exclusive measurements allow, in principle, to determine the magnetic moments of charmed baryons.
\end{abstract}

The purpose of this paper is to study the associative photoproduction of charmed particles on nucleon. Among the simplest reactions of open charm production, $\gamma+N \rightarrow \mathcal{B}_{c}+\bar{D}$, with $\mathcal{B}_{c}=\Lambda_{c}$ or $\Sigma_{c}$, the reaction $\gamma+p \rightarrow \Lambda_{c}^{+}+\overline{D^{0}}$ has the lowest threshold. The experimental data about charm photoproduction concern mainly inclusive production of $D\left(D^{*}\right)$-mesons or $\Lambda_{c}\left(\Sigma_{c}\right)$ - baryons at high energies, very far from threshold. The lowest photon energy where charm production was observed is $20 \mathrm{GeV}$, at SLAC [1] , with an indirect estimation of the corresponding cross section. Up to now no exclusive measurement exists. Photoproduction processes involving charmed particles are experimentally accessible with a photon beam of energy over $10 \mathrm{GeV}$. The possibility of a systematic study of these reactions in the treshold region at future machines as ELFE [2] or at the Jefferson Laboratory (JLab) electron accelerator, after the planned upgrade [3], makes this problem very actual.

The photon-gluon fusion, $\gamma+g \rightarrow c+\bar{c}$, is considered as the most probable mechanism of inclusive photoproduction of charmed particles at high energy. In its different versions (adding, for example, NLO contributions), the inclusive spectra of $D^{*}$ and $D$-mesons [4] can be satisfactorily reproduced. However this mechanism predicts $\bar{D} / D$ as well as $\overline{\Lambda_{c}} / \Lambda_{c}$ symmetry, in contradiction with the experimental data [5,6]. Open charm photoproduction can induce, at least partly, the observed asymmetry. The standard view of inclusive charm photoproduction, based on $\gamma+g-$ fusion, with subsequent fragmentation of $c+\bar{c}$ into charmed particles can not be directly applied to the description of the exclusive reactions, in any kinematical conditions, and in particular in the threshold region. Also other approaches, based on VDM [7] or QCD [8], which may reproduce the total inclusive cross section, do not give a simple picture of exclusive processes.

We will use a formalism based on an effective Lagrangian approach (ELA), extrapolating a method which is well known in the domain of the photoproduction of $\pi$ - and $K$-mesons. We will consider the photoproduction of pions, $\gamma+N \rightarrow N+\pi$; of strange particles, $\gamma+N \rightarrow \Lambda+K$; and of open charm, $\gamma+N \rightarrow \mathcal{B}_{c}+\bar{D}$, as the same class of reactions $\gamma+N \rightarrow \mathcal{B}+\mathcal{P}$ where $\mathcal{B}$ is a baryon, with spin $\mathcal{J}$ and parity $P$ equal to $\mathcal{J}^{P}=1 / 2^{+},\left(\mathcal{B}=N, \Lambda, \Sigma, \Lambda_{c}\right.$, or $\Sigma_{c}$ ) and $\mathcal{P}$ is the corresponding pseudoscalar meson $(\mathcal{P}=\pi, \eta, K, D)$. In our knowledge, there is no principal restriction on the mass of the $\mathcal{P}$-meson for the applicability of ELA. Note in this respect, an interesting scaling for the masses of pseudoscalar mesons: $m_{K} / m_{\pi} \simeq m_{D} / m_{K} \simeq 3.6$.

The application of ELA to exclusive charm photoproduction has the following advantages:

- the transparent physical content of the considered mechanisms;

- limited number of parameters with a definite physical meaning: interaction constants and magnetic moments of charmed baryons;

- theoretical predictions for parameters such as magnetic moments of charmed baryons can be used in the numerical calculations;

- the outputs of the model are the absolute value of the differential cross section and all polarization observables, in the near threshold region.

Such approach has been recently used in the studies of diferent processes involving charm particles, as $J / \psi+\pi(\rho) \rightarrow$ $D+\bar{D}\left(D^{*}\right)$. or $J / \psi+N \rightarrow \Lambda_{C}+\bar{D}[9,10,12,11$. The cross sections for these reactions have been calculated in the ELA formalism, considering the pole diagrams (with $D, D^{*}$ or $\Lambda_{C}$ exchanges), in relation with the study of the mechanisms responsible for the $J / \psi$ suppression in high energy nuclei collisions.

*Permanent address: National Science Center KFTI, 310108 Kharkov, Ukraine 
The present analysis should be considered as a starting point in the discussion of the possible mechanisms which play the most important role in the threshold region [13]. The predictive power of such approach should be useful in planning the possible future measurements of cross sections or polarization observables.

We found a large sensitivity of the observables to different fundamental parameters: as an example, the absolute value of the differential cross section depends mainly on the interaction constant in the vertex $N \mathcal{B}_{c} D$, while the magnetic moments of the charmed baryons mostly affect the beam asymmetry. The magnetic moments of charmed particles can be calculated in different approaches, from quark models to ChPT+HQET considerations, 14 17]. In this respect the $\Lambda_{c}$ is very interesting: the light quarks, here, are in a configuration with zero total spin, so the $\Lambda_{c}$ magnetic moment is due only to the the magnetic moment of the heavy $c$-quark [15]. There is no experimental information about the magnetic moments of the charmed baryons. The standard methods used for usual hyperons can not be applied here, due to the shorter time of life for $\mathcal{B}_{c}$. In principle the method of bending these particles in crystals can be used [18]. The processes $\gamma+N \rightarrow \mathcal{B}_{c}+\bar{D}$ can be considered a parallel and independent way.

According to the lines given by a previous work [13, which we update and complete, we calculate the differential cross section and the excitation function for the process $\gamma+p \rightarrow \Lambda_{c}^{+}+\overline{D^{0}}$. We calculate the beam asymmetry and discuss the sensitivity of the different observables to the magnetic moment of the $\Lambda_{c}^{+}$.

The spin structure for the amplitude of the processes $\gamma+N \rightarrow \mathcal{B}_{c}+\bar{D}$ can be written in a general form (in the CMS of the considered reaction):

$$
\mathcal{F}=i \vec{\sigma} \cdot \vec{e} f_{1}+\vec{\sigma} \cdot \hat{\vec{q}} \vec{\sigma} \cdot \hat{\vec{k}} \times \vec{e} f_{2}+i \vec{e} \cdot \hat{\vec{q}} \vec{\sigma} \cdot \hat{\vec{k}} f_{3}+i \vec{\sigma} \cdot \hat{\vec{q}} \vec{e} \cdot \hat{\vec{q}} f_{4}
$$

where $\vec{e}$ is the photon polarization three-vector, $\hat{\vec{k}}$ and $\hat{\vec{q}}$ are the unit vectors along the three-momentum of $\gamma$ and $\bar{D}$. The differential cross section is given by:

$$
\frac{d \sigma}{d \Omega}=\frac{q}{k}\left(E_{1}+m\right)\left(E_{2}+M\right) \frac{B+A \sin ^{2} \vartheta}{128 \pi^{2} s}
$$

with

$$
\begin{gathered}
A=\left|f_{3}\right|^{2}+\left|f_{4}\right|^{2}+2 \mathcal{R} e f_{2} f_{3}^{*}+2 \mathcal{R} e\left(f_{1}+\cos \vartheta f_{3}\right) f_{4}^{*}, \\
B=2\left(\left|f_{1}\right|^{2}+\left|f_{2}\right|^{2}-2 \cos \vartheta \mathcal{R} e f_{1} f_{2}^{*}\right),
\end{gathered}
$$

where $E_{1}\left(E_{2}\right)$ and $m(M)$ are the energy and the mass of $N\left(\mathcal{B}_{c}\right)$, respectively, and $\vartheta$ is the center of mass angle of the $D$-meson production (with respect to the direction of the incident photon). In case of a linearly polarized photon beam, the beam asymmetry $\Sigma$ can be determinated as:

$$
\Sigma=\frac{d \sigma_{\perp} / d \Omega-d \sigma_{\|} / d \Omega}{d \sigma_{\perp} / d \Omega+d \sigma_{\|} / d \Omega}=\frac{-A \sin ^{2} \vartheta}{B+A \sin ^{2} \vartheta}
$$

The amplitudes $f_{i}, i=1-4$, have to be calculated in the framework of some model. We consider here the pole contributions in the $s-, t-$ and $u$-channels (Fig. 1), so $f_{i}=f_{i, s}+f_{i, t}+f_{i, u}$.

A discussion of the ELA applied to charm particles, with broken SU(4) symmetry, can be found in literature [9]. In the framework of this formalism, one obtains the following expressions for the matrix element, corresponding to the $s-, t-$ and $u$-contributions (for the pseudoscalar meson-baryon vertex):

$$
\begin{gathered}
\mathcal{M}_{s}=e \frac{g_{N \mathcal{B}_{c} D}}{s-m^{2}} \bar{u}\left(p_{2}\right) \gamma_{5}\left(\hat{k}+\hat{p}_{1}+m\right)\left[Q_{N} \hat{\epsilon}-\frac{\hat{\epsilon} \hat{k}}{2 m} \kappa_{N}\right] u\left(p_{1}\right), \\
\mathcal{M}_{u}=e \frac{g_{N \mathcal{B}_{c} D}}{u-m^{2}} \bar{u}\left(p_{2}\right)\left(Q_{c} \hat{\epsilon}-\frac{\hat{\epsilon} \hat{k}}{2 M} \kappa_{C}\right)\left(\hat{p}_{2}-\hat{k}+M\right) \gamma_{5} u\left(p_{1}\right), \\
\mathcal{M}_{t}=2 e Q_{D} \frac{g_{N \mathcal{B}_{c} D}}{t-M_{D}^{2}} \hat{\epsilon} \cdot \hat{q} \bar{u}\left(p_{2}\right) \gamma_{5} u\left(p_{1}\right),
\end{gathered}
$$

where $k, q, p_{1}$ and $p_{2}$ are the four momenta of $\gamma, D, N$ and $\Lambda_{c}, \epsilon(\epsilon \cdot k=0)$ is the four vector of the photon polarization, $s=\left(k+p_{1}\right)^{2}, u=\left(k-p_{2}\right)^{2}, t=(k-q)^{2}$ are the Mandelstam variables, $m\left(Q_{N}\right), M\left(Q_{c}\right)$ and $M_{D}\left(Q_{D}\right)$ are the 
masses (electric charges) of the nucleon, the charmed baryon and the $D$-meson, respectively, $g_{N \mathcal{B}_{c} D}$ is the coupling constant for the vertex of nucleon-charmed baryon- $D$ meson interaction; $\kappa_{N}$ and $\kappa_{c}$ are the nucleon and charmed baryon anomalous magnetic moment $\left(\kappa_{N}=1.79(-1.91)\right.$ for $\left.p(n)\right)$.

From these formulas we can derive the following expressions for the scalar amplitudes $f_{i}$. s-channel:

$$
\begin{gathered}
f_{1, s}=e \frac{g_{N \mathcal{B}_{c} D}}{W+m}\left[Q_{N}-(W-m) \frac{\kappa_{N}}{2 m}\right], \\
f_{2, s}=e \frac{g_{N \mathcal{B}_{c} D}}{W+m}\left[-Q_{N}-(W+m) \frac{\kappa_{N}}{2 m}\right] \frac{|\vec{q}|}{E_{2}+M}, \\
f_{3, s}=f_{4, s}=0, \frac{e^{2}}{4 \pi}=\alpha \simeq \frac{1}{137},
\end{gathered}
$$

where $W=\sqrt{s}$, is the total energy, related to the $\gamma$-energy in the laboratory system by $s=m^{2}+2 E_{\gamma} m$. u-channel:

$$
\begin{gathered}
f_{1, u}=e \frac{g_{N \mathcal{B}_{c} D}}{u-M^{2}}\left\{Q_{c}(W-M)-\frac{\kappa_{c}}{2 M}\left[t-M_{D}^{2}+(W-m)(W+m-2 M)\right]\right\} \\
f_{2, u}=-e \frac{g_{N \mathcal{B}_{c} D}}{u-M^{2}} \frac{|\vec{q}|}{E_{2}+M}\left\{Q_{c}(W+M)+\frac{\kappa_{c}}{2 M}\left[t-M_{D}^{2}+(W+m)(W-m+2 M)\right]\right\} \frac{W-m}{W+m} \\
f_{3, u}=e \frac{g_{N \mathcal{B}_{c} D}}{u-M^{2}}|\vec{q}| \frac{W-m}{W+m}\left[2 Q_{c}+\kappa_{c} \frac{W+m}{M}\right] \\
f_{4, u}=\frac{g_{N \mathcal{B}_{c} D e}}{u-M^{2}}\left(E_{2}-M\right)\left[-2 Q_{c}+\kappa_{c} \frac{W-m}{M}\right]
\end{gathered}
$$

\section{t-channel:}

$$
\begin{gathered}
f_{1, t}=f_{2, t}=0 \\
f_{3, t}=-2 e Q_{D} \frac{g_{N \mathcal{B}_{c} D}}{t-M_{D}^{2}}|\vec{q}| \frac{W-m}{W+m} \\
f_{4, u}=2 e Q_{D} \frac{g_{N \mathcal{B}_{c} D}}{t-M_{D}^{2}}\left(E_{2}-M\right) .
\end{gathered}
$$

The angular distribution and the beam asymmetry for the process $\gamma+p \rightarrow \Lambda_{c}^{+}+\overline{D^{0}}$ are reported on Fig. 2 as functions of $\vartheta$. The full line represents the sum of all contributions, the Born $s$-channel term is given by the dashed line and the Born $u$-channel term by the dotted line. The $t$ - channel diagram does not play any role for this reaction in the present model, as $Q_{D}=0$. Even in the case when the contribution of one diagram is negligible its interference with the other terms can largely affect the total result: a large effect of the $s$ - $u-$ interference may appear in the differential cross section (Fig. 2).

The plotted differential cross section is divided by $g_{N D \Lambda_{c}}^{2}$, for which we do not have (theoretical or experimental) precise indications.

The behavior of the $\Sigma$-asymmetry results from the difference in the spin structure of the $s$ and $u$ - channel contributions to the matrix element for $\gamma+p \rightarrow \Lambda_{c}+\overline{D_{0}}$. The $s-$ channel diagram (mainly characterized by s-wave $\Lambda_{c} D$-production with a small off-mass shell admixture of p-wave) can not induce nonzero $\Sigma$-asymmetry, contrary to the $u$-channel contribution, which contains the majority of the multipole amplitudes, even in the threshold region, and is responsible for the positive value of $\Sigma$, in the whole angular domain. However the final result is essentially driven by the $s-u$-interference, which is negative, compensating the positive $u$-channel contribution. 
In order to illustrate the sensitivity of this reaction to the $\Lambda_{c}$ magnetic moments, in Fig. 3 we show, as a function of $\mu_{\Lambda_{c}}$, the dependence of the, taken at $\vartheta=\pi / 2$ and of the ratio of the differential cross section for $\vartheta=\pi$ and $\vartheta=0$ (backward-forward asymmetry). Both these quantities show a characteristic dependence on $\mu_{\Lambda_{c}}$, in the region $\mu_{\Lambda_{c}} \leq 1$, being almost independent on $\mu_{\Lambda_{c}}$ for $\mu_{\Lambda_{c}} \geq 1$. The asymmetry $\Sigma(\pi / 2)$ changes sign, for $\mu_{\Lambda_{c}} \simeq 1$.

The sensitivity of the absolute cross section to $\mu_{\Lambda_{c}}$ appears explicitely at threshold, where only the amplitude $f_{1}$ is present and can be written as:

$$
f_{1} \simeq 1-\frac{W-m}{2 M_{D}}\left(\mu_{\Lambda_{c}}-1+\kappa_{p} \frac{M}{m}\right) \propto 1+0.46 \mu_{\Lambda_{c}} .
$$

The excitation functions for all the considered reactions, with magnetic moments as reported in the Table, are shown in Fig. 4: $\gamma+p \rightarrow \Lambda_{c}^{+}+\overline{D^{0}}$ (thick solid line), $\gamma+p \rightarrow \Sigma_{c}^{++}+D^{-}$(dashed line), $\gamma+p \rightarrow \Sigma_{c}^{+}+\overline{D^{0}}$ (dotted line), $\gamma+n \rightarrow \Lambda_{c}^{+}+D^{-}$(dot-dashed line), $\gamma+n \rightarrow \Sigma_{c}^{+}+D^{-}$(solid line), $\gamma+n \rightarrow \Sigma_{c}^{0}+\overline{D^{0}}$ (thick dot-dashed line). The calculation for the reaction $\gamma+p \rightarrow \Lambda_{c}^{+}+\overline{D^{0}}$, using the magnetic moment suggested by [16] is reported as a thick dashed line.

These calculations reproduce satisfactorily the measurements at high energy (on proton target) but overestimate the measurement at the lowest energy, from [1]. The interest of such a comparison with the experimental is to give an estimation of the upper limit of the constant $g_{N \mathcal{B}_{c} D}$, here taken as unity.

In the present model, the cross section for the process $\gamma+n \rightarrow \Sigma_{c}^{0}+D^{0}$ is larger when compared with the other processes, due to the fact that the magnetic moments of $n$ and $\Sigma_{C}^{0}$ contribute coherently in the $s-$ and $u-$ channel.

Note that the possible T-odd polarization observables have to be identically zero, in the framework of the considered model, in any kinematical conditions, for any reaction $\gamma+N \rightarrow \mathcal{B}_{c}^{+}+\bar{D}$.

In conclusion, we have calculated the differential cross section and the beam asymmetry for the exclusive photoproduction of charmed particles near threshold, $\gamma+N \rightarrow \mathcal{B}_{c}+\bar{D}$. We have studied the effects of the magnetic moments of charmed baryons and of the coupling constant for the $N \mathcal{B}_{c} D$ vertex and discussed the possibility to determine these quantities, which are not known experimentally. We have shown that the absolute value of the cross section depends mainly on the interaction constant in the vertex $N \mathcal{B}_{c} D$. On the other hand, the angular dependence and even the sign of the beam asymmetry is largely affected by the value of the magnetic moment of the charmed baryon.

[1] K. Abe et al., Phys. Rev. D 30 (1984) 1.

[2] J. Arvieux and E. de Sanctis The ELFE Project, (Ed. Compositori, Bologna, 1992).

[3] See the website http://www.jlab.org/ gen/charm/.

[4] C. Adloff et al., H1 Coll., Nucl. Phys. B 545 (1999) 21;

J. Breitwag et al., ZEUS Coll., Eur. Phys. Journ. C 6(1999) 67; C 12 (2000) 35.

[5] J. C. Anjos et al., Tagged Photon Spectrometer Coll., Phys. Rev. Lett. 62 (1989) 513.

[6] P. L. Frabetti et al., E687 Coll., Phys. Lett. B 370 (1996) 222.

[7] H. Fritsch, Phys. Lett. B 67, (1977) 217;

H. Fritsch and K. H. Streng, Phys. Lett. B 78 (1978) 447.

[8] V. A. Novikov et al., Nucl. Phys. B 136 (1978) 12.

[9] S. G. Matinyan and B. Muller, Phys. Rev. C 58 (1998) 2994.

[10] K. L. Haglin, nucl-th/9907034 (1999).

[11] Z. Liu and C. M. Ko nucl-th/9912046 (1999)

[12] Z. Liu, C. M. Ko and B. Zhang, Phys. Rev. C 61 (2000) 024904.

[13] M. P. Rekalo, Ukr. Fiz. Journ. 22 (1977) 1602.

[14] A. L. Choudhury and V. Joshi, Phys. Rev. D13, 3120 (1976).

[15] D.-P. Min, Proc. Int. Workshop 'Baryon Spectroscopy and the structure of the Nucleon (Saclay, 23-25 Sept. 1991) p. 138.

[16] M. J. Savage, Phys. Lett. B 326, (1994) 303.

[17] M. C. Banuls et al., Phys. Rev. Lett. D 61 (2000) 074007.

[18] D. Chen et al., E761 Coll., Phys. Rev. Lett. 69 (1992) 3286.

[19] M . Alvarez et al., NA 14/2 Coll. Z. Phys. C 60 (1993) 53.

[20] J.J. Aubert et al., EMC Coll. Nucl. Phys B 213 (1983) 31.

[21] D. Aston et al., WA4 Coll. Phys. Lett. B 94 (1980) 113.

[22] M. J. Adamovich et al., Photon Emulsion Coll., Phys. Lett. B 187 (1987) 437. 


\begin{tabular}{|c|c|c|c|c|c|}
\hline Reaction & Threshold $[\mathrm{GeV}]$ & $\mu_{\Lambda_{c}, \Sigma_{c}}\left[\mu_{N}\right]$ & $\kappa_{c}\left[\mu_{N}\right]$ & $M_{\mathcal{B}_{c}}[\mathrm{GeV}]$ & $M_{D}[\mathrm{GeV}]$ \\
\hline$\gamma+p \rightarrow \Sigma_{c}^{++}+D^{-}$ & 9.4856 & 1.86 & -0.14 & 2.4528 & 1.8693 \\
\hline $\bar{\gamma} \gamma+n \rightarrow \Lambda_{c}^{+}+D^{-}$ & 8.7139 & 1.86 & $\overline{0.86}$ & 2.2849 & $\overline{c 1.8693}$ \\
\hline $\bar{c} \gamma+n \rightarrow \Sigma_{c}^{+}+D^{-}$ & 9.4750 & 0 & -1 & 2.4536 & 1.8693 \\
\hline
\end{tabular}

TABLE I. Table of reactions and constants.

FIG. 1. Feynman diagrams calculated for the process $\gamma+N \rightarrow \mathcal{B}_{c}+\bar{D}$ : (a)- $s$-channel, (b)- $u$-channel, (c)- $t$-channel.

FIG. 2. Differential cross section and beam asymmetry as a function of the $D$-meson cms angle, $\vartheta$ : full calculation (solid line), Born $s$-channel (dashed line), and Born $u$-channel (dotted line).

FIG. 3. Asymmetry calculated at $\vartheta=\pi / 2$ (top) and ratio of the cross section for $\vartheta=\pi$ and $\vartheta=0$ (bottom) as functions of the $\Lambda_{c}$ magnetic moment.

FIG. 4. Sample of existing experimental data from 伍] (circle), 19] (square), 20] (triangles), [21] (reversed triangle), [5] (open circle), 22] (open square). The different lines show the calculations for the different reactions (see text and Table), asssuming the interaction constant $g_{N \mathcal{B}_{c} D}=1$. 

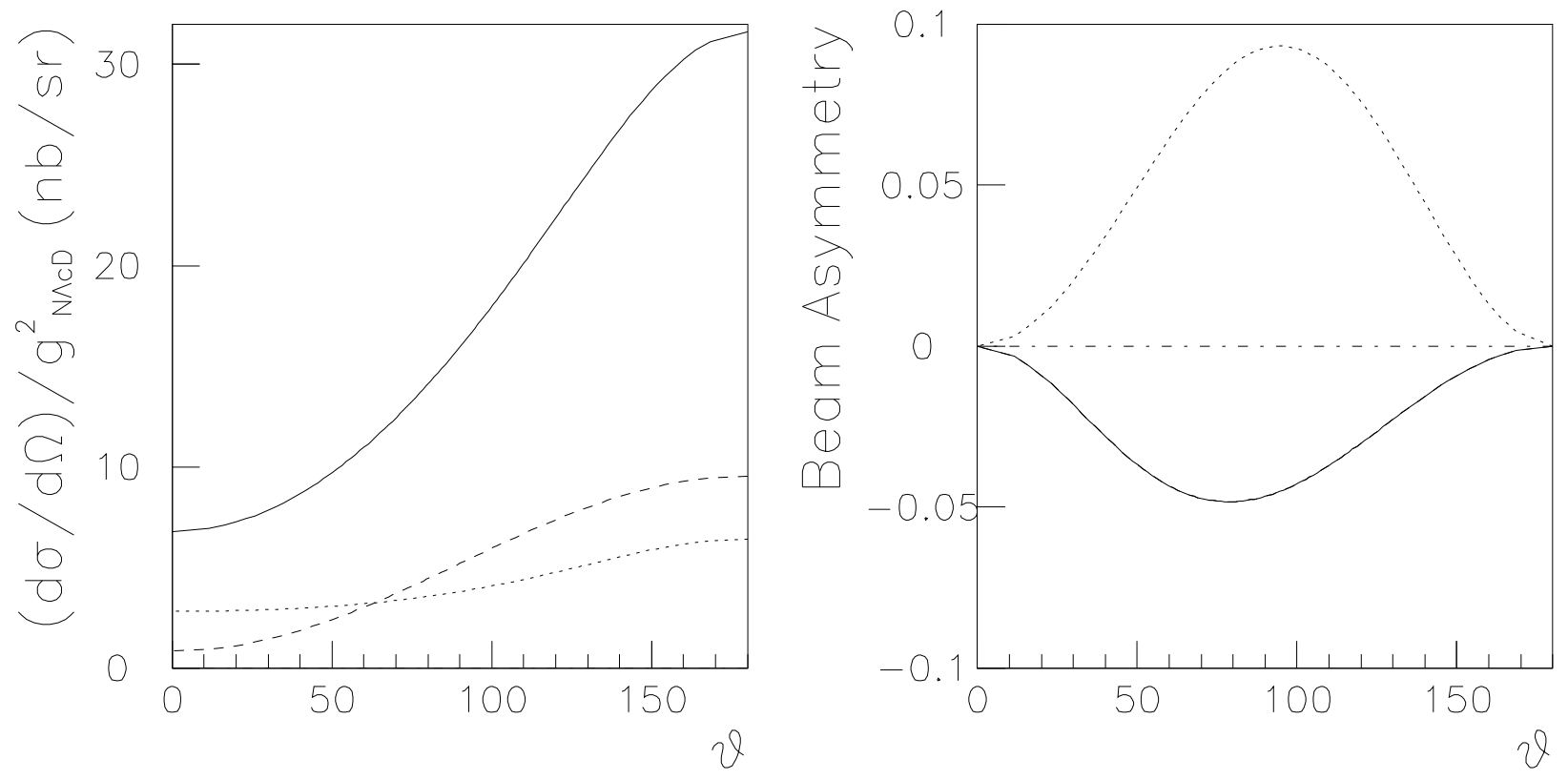


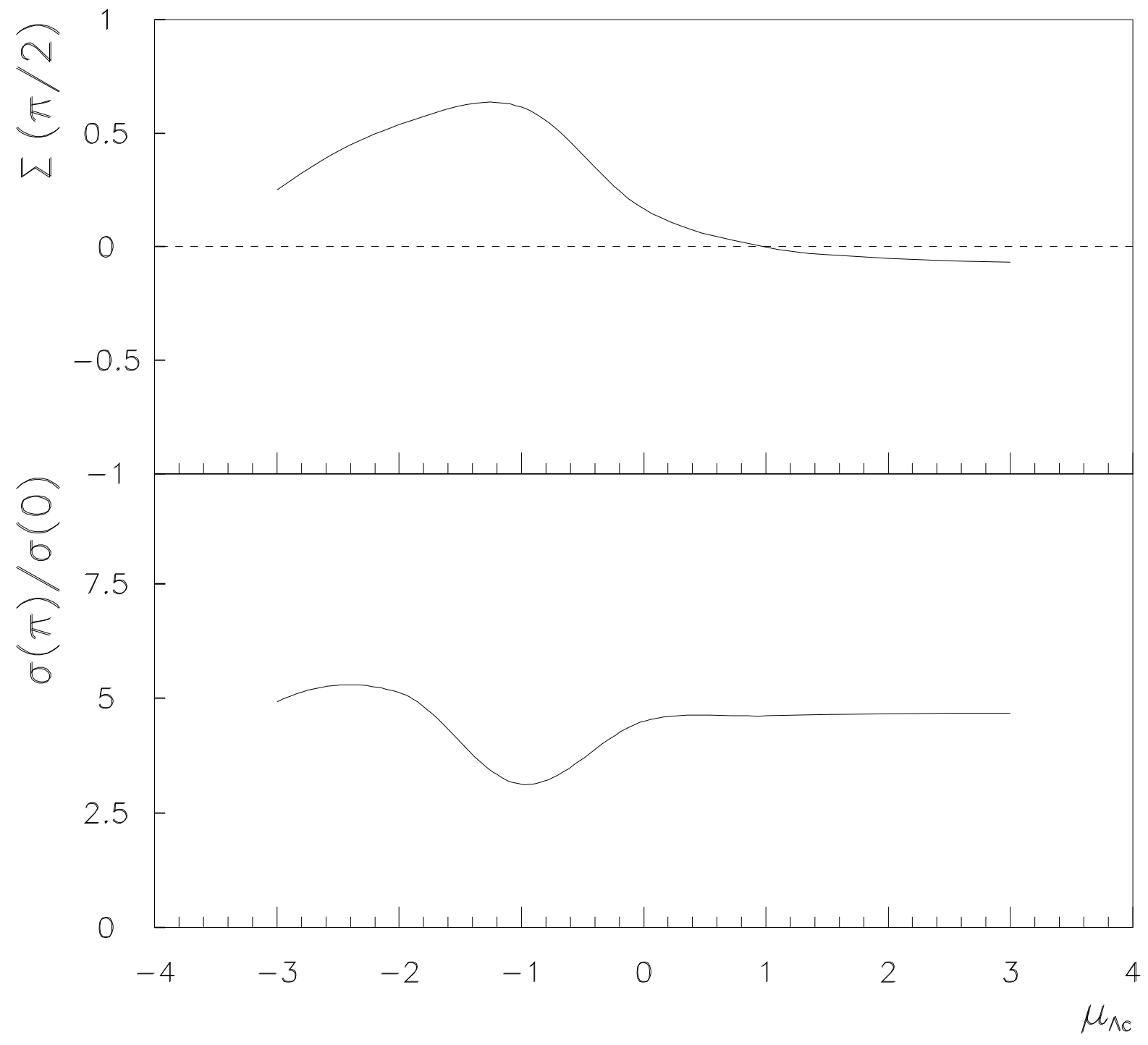




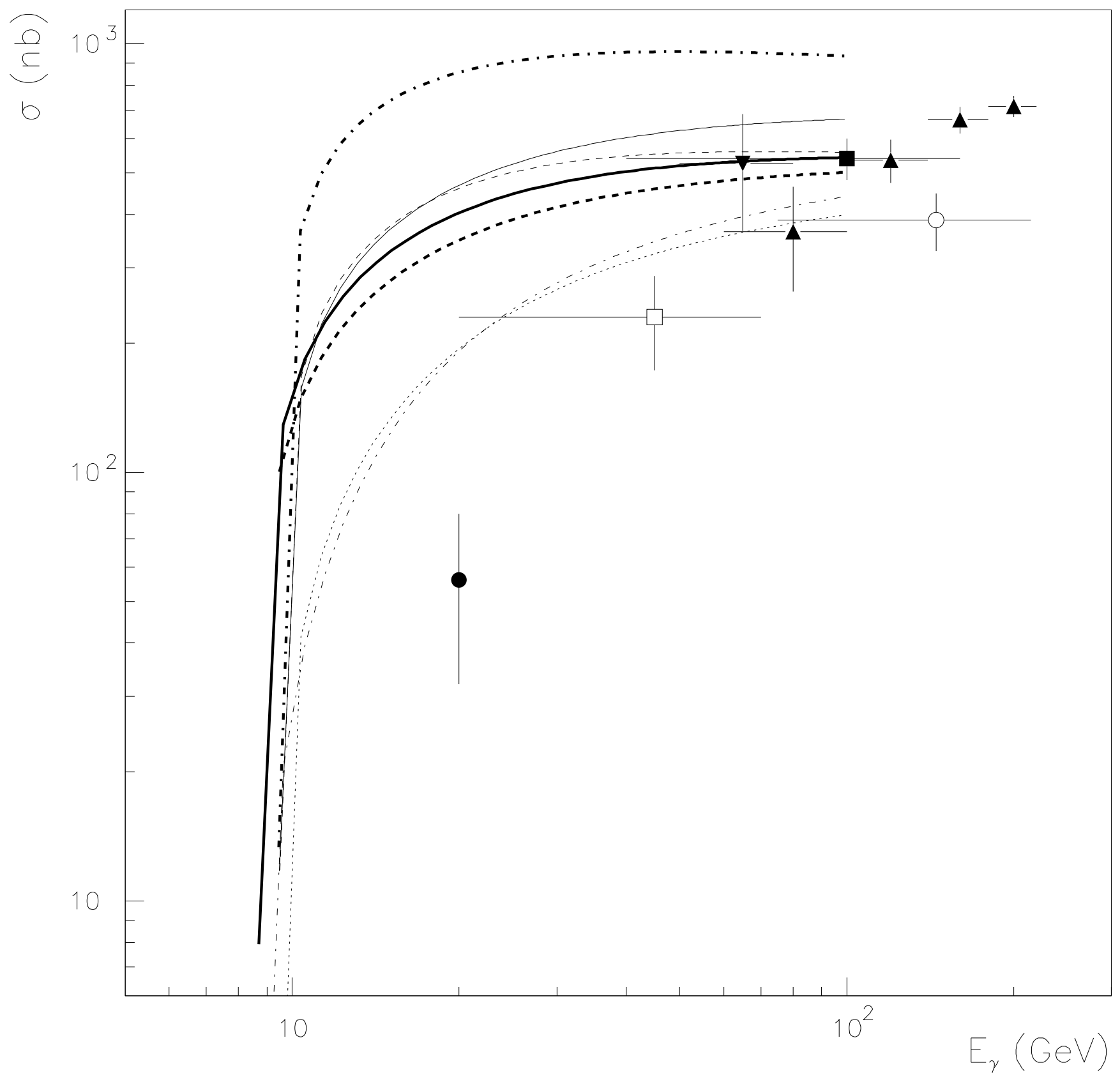

